\title{
ANDARES CURRICULARES EN AMÉRICA LATINA
}

\author{
CURRICULAR STRIDES ON LATIN AMERICA
}

\author{
Ángel Díaz-Barriga1 \\ Doctor en Pedagogía \\ Investigaciones sobre la Universidad y la Educación \\ Universidad Nacional Autónoma de México \\ adbc49@gmail.com
}

\begin{abstract}
Resumen: El campo del curriculum se ha conformado en una disciplina compleja en el ámbito de las ciencias sociales. De alguna forma, es una disciplina contemporánea, ya que surge en el proceso de consolidación de los sistemas educativos nacionales $\mathrm{y}$, en las transformaciones sociales y científicas gestadas con la industrialización del siglo XX. En sus orígenes es una disciplina que surge en el conflicto entre atender los procesos de los sujetos y las necesidades de las instituciones y de los sistemas sociales.

El campo del curriculum se desarrolla en América Latina desde dos vertientes: la que impulsan los ministerios de educación, apoyados en la ideología de la OCDE y de su prueba PISA impulsando logro de resultados homogéneos y la que desarrollan algunos (pocos) investigadores en el campo del currículo con relación a alguna de las líneas diversas de debate.

Otro problema emerge con el empleo de la perspectiva eficientista-curricular el desplazamiento genérico del pensamiento pedagógico en la región. Los sujetos de la educación dejaron de importar, lo relevante es impulsar que todos logren aprendizajes similares. La homogeneidad por encima de todo.

Imposible dar cuenta de todo ello en un ensayo, pero se hace necesario ir avanzando en este tipo de indagaciones.
\end{abstract}

Palabras clave: Campo del curriculum, curriculum en América latina, corrientes del campo curricular, curriculum en la academia

Abstract: The field of the curriculum has become a complex discipline in the field of social sciences. In some way, it is a contemporary discipline, since it arises in the process of consolidation of national educational systems and, in the social and scientific transformations gestated with the industrialization of the 20th century. In its origins, it is a discipline that arises in the conflict between attending to the processes of the subjects and the needs of institutions and social systems.

The curriculum field is developed in Latin America from two aspects: the one promoted by the ministries of education, supported by the ideology of the OECD and its PISA test, promoting the achievement of homogeneous results, and the one developed by some (few) researchers in the field. of the curriculum in relation to any of the various lines of debate.

${ }^{1}$ Investigador emérito del IISUE-UNAM. México

Departamento de Educación - Facultad de Ciencias Sociales - Universidad de Chile 
Another problem emerges with the use of the efficiency-curricular perspective, the generic displacement of pedagogical thought in the region. The subjects of education no longer mattered, the relevant thing is to encourage everyone to achieve similar learning. Homogeneity above all else.

It is impossible to account for all this in an essay, but it is necessary to move forward in this type of investigation.

Key Words: Curriculum field, curriculum in Latin America, currents of the curricular field, curriculum in the academy

\section{INTRODUCCIÓN}

El campo del curriculum se ha conformado en una disciplina compleja en el ámbito de las ciencias sociales. De alguna forma es una disciplina contemporánea, ya que surge en el proceso de consolidación de los sistemas educativos nacionales $\mathrm{y}$, en las transformaciones sociales y científicas gestadas con la industrialización del siglo XX. En sus orígenes es una disciplina que surge en el conflicto entre atender los procesos de los sujetos y las necesidades de las instituciones y de los sistemas sociales.

Más allá del conflicto el debate eficientista que dominó al campo. Esta fue la versión en su expresión técnica con la que llegó en los años sesenta y setenta del siglo pasado a América Latina, más allá de que en esa época en el país de origen se estuviera llevando a cabo una ruptura entre el pensamiento técnico eficientista. Este papel lo jugaron los desarrollos entorno al concepto curriculum oculto; a la visión del curriculum como campo moribundo; así como la construcción que se denominó la teoría deliberativa y; en este mismo periodo el surgimiento de la perspectiva reconceptualista (o de reconceptualización curricular) de Pinar y la visión crítica marxista.

Con independencia de lo intenso del debate curricular pos-tecnicista, la realidad en América Latina ha sido que es este debate el que ha dominado la región. Los principales impulsores del mismo son las autoridades educativas pues la visión tecnicista ha sido muy oportuna para su necesidad de homogeneizar los resultados educativos. Ciertamente esta se ha modernizado cada cierto tiempo: curriculum flexible, enfoque de competencias en el currículo, incorporación de tecnologías e innovación curricular. Cambios cosméticos para mantener el predominio de la perspectiva técnico-eficientista, con su modernización necesaria.

De esta manera el campo se desarrolla en América Latina desde dos vertientes: la que impulsan los ministerios de educación, apoyados en la ideología de la OCDE y de su prueba PISA impulsando logro de resultados homogéneos y la que desarrollan algunos (pocos) investigadores en el campo del currículo con relación a alguna de las líneas diversas de debate.

Otro problema emerge con el empleo de la perspectiva eficientista-curricular el desplazamiento genérico del pensamiento pedagógico en la región. Los sujetos de la 
educación dejaron de importar, lo relevante es impulsar que todos logren aprendizajes similares. La homogeneidad por encima de todo.

Imposible dar cuenta de todo ello en un ensayo, pero se hace necesario ir avanzando en este tipo de indagaciones.

\section{UNA PERSPECTIVA SOBRE ALGUNOS AVATARES DEL CAMPO EN LA REGIÓN}

Un tema por demás ambicioso es intentar dar cuenta de cómo ha evolucionado el campo del currículo en una región que en ocasiones consideramos con rasgos comunes, pero en donde cada país y dentro del mismo cada comunidad académica ha asumido el campo curricular.

No sólo hay rasgos específicos de cada país en América Latina, que tienen que ver con las condiciones generales de su población, sus semejanzas y diferencias culturales, su diverso grado de desarrollo. Condiciones de las cuáles las tradicionales clasificaciones económicas no dan cuenta de ello, sea que se les llame como se les consideró en los años cincuenta del siglo pasado países en desarrollo, posteriormente se les llamó países del tercer mundo, y, algunos de ellos han sido reconocidos como economías emergentes. Por el contrario, son países caracterizados por una gran desigualdad social, con porcentajes significativos de habitantes en pobreza y en pobreza extrema, al mismo tiempo que sectores pequeños de la población concentran las ganancias económicas. La desigualdad social es un rasgo característico de América Latina.

La región ha sido, quizá como ninguna del mundo, objeto de una intervención directa o indirecta de recomendaciones que emanan de diversos organismos internacionales. No todos ellos tienen la misma presencia, ni el mismo impacto en los diferentes países, quizá hasta se puede rastrear en algunos casos cómo el impacto de un organismo es reemplazado por otro. Desde fines de los años ochenta del siglo pasado, en parte como consecuencia de la llamada década perdida, América Latina se convirtió en un espacio para impulsar diversas propuestas económicas, sociales, de salud y educativas que emanan de tales organismos. Algunos claramente vinculados a los grandes capitales, cuyo rasgo característico cuando aterrizan su proyecto en un modelo pedagógico es ver a la educación como una mercancía en donde hay que impulsar la homogeneidad de los resultados educativos en pro de políticas centradas en la eficiencia en el trabajo escolar. En este contexto, los maestros fueron considerados los responsables de los llamados logros educativos. Y paulatinamente se fue estableciendo un conjunto de programas bajo la denominación "evaluación" como un elemento garante del logro de la calidad. Los años noventa del siglo pasado y primera década de este siglo, los sistemas de acreditación de instituciones y planes de estudios, llamados programas, una copia clara del anglisismo de origen), fueron desplazando diversas prácticas el campo curricular.

Por ejemplo: la evaluación curricular, perspectiva que nunca logró consolidarse y que prácticamente no existe en el debate sajón, particularmente en el estadounidense. (DíazBarriga, 2009). Dado que, si bien se generalizó la visión técnica de la construcción de planes 
de estudio, en este paquete no venía incluida la evaluación del currículo, innecesaria en un país donde desde principios del siglo XX se había establecido la acreditación (Ed-Kawas, 2001). El tema evaluación del currículo empezaba a conformarse en la región con el trabajo de Lewy (1976), de Alba (1991), Ruíz (2001) y Valenzuela (2005). La evaluación del currículo realizada desde una perspectiva pedagógica sucumbiría a los instrumentos formales de la información que se solicita en los documentos que se exigen a una institución en el proceso de acreditación denominados "autoevaluación", que en realidad son informes cuantitativos de un conjunto de variables formales: número de publicaciones, número de profesores de tiempo completo, lista de investigaciones, mecanismos de ingreso para los estudiantes, trayectoria académica de los estudiantes, número de libros en la biblioteca, fechas en las que se han realizado reformas curriculares, entre otras. Lo formal sobre el proceso pedagógico. Convirtiendo este informe en el documento base para las entrevistas que realizan los evaluadores. En varios países los resultados de estas entrevistas son integrados a una lista de cotejo para su posterior manejo cuantitativo. La evaluación curricular sencillamente no logró consolidarse en la región y fue ampliamente desplazada por los diversos mecanismos de acreditación.

Lentamente, pero en una especie de finalidad común, se fueron estableciendo proyectos educativos y curriculares que, tras defender el mejoramiento y la calidad de la educación, fueron implantando una ideología eficientista en el trabajo escolar. Lo que se realizó a través del modelo taxonómico para clasificar aprendizajes, propuesto por Benjamin Bloom y sus colaboradores en los años cincuenta del siglo pasado, así como recientemente por la incorporación del enfoque de competencias impulsado a principios de este siglo.

Bajo la perspectiva de que lo que no se mide no se puede mejorar, los aprendizajes se redactaron en términos de objetivos de comportamientos, de competencias, de aprendizajes clave, de núcleos de aprendizaje, siempre en la perspectiva de poder ser medidos a través de exámenes a gran escala. Lo que significó no sólo abandonar la tarea de formación y desarrollo personal como objeto del trabajo pedagógico (Horlacher, 2015), sino reducir el aprendizaje a algo observable y comprobable, en el lenguaje actual presentar aprendizajes logrados o evidencias de aprendizaje. De manera un tanto incierta se empezaron a aplicar pruebas nacionales a gran escala, hasta que, al inicio del siglo, ya operando como el superministerio de educación global, la OCDE propuso y, los gobiernos nacionales poco a poco han ido aceptando la prueba: PISA, para comparar los conocimientos y habilidades necesarios para la vida en la versión del año 2000 y, desde 2003 las competencias para desempeñarse en una ciudadanía global.

De forma paulatina se fue impulsando en la región la idea de que es el docente el principal y, en ocasiones único, responsable de los resultados de aprendizaje que obtienen sus alumnos, poco a poco se fue instaurando un sistema llamado de evaluación docente, que fue incapaz de trabajar con la complejidad de procesos que se generan en el aula. Se optó por concebir la evaluación docente como un sistema de medición de conocimientos curriculares, disciplinarios y pedagógicos, para lo cual también se diseñaron diversos instrumentos de medición, como exámenes a gran escala y la presentación de portafolio de evidencias, se desconoció de facto lo situacional de cada relación pedagógica y la diversidad de decisiones profesionales que un docente toma en cada sesión de trabajo con sus estudiantes. Con estas 
mediciones se obtuvieron clasificaciones de docentes de acuerdo a los puntajes obtenidos, que fundamentalmente contribuyeron al desprestigio social de la profesión. Las pruebas ideadas para tal efecto en ningún momento se refirieron a la práctica en el aula, sino que impusieron una visión muy limitada sobre todo de saberes pedagógicos, ignorando los saberes que emanan de la experiencia y el sentido utilitario que tienen los saberes docentes para el trabajo con los alumnos. (Tardif, 2010)

\section{EN UN CONTEXTO DE CONVULSIÓN POLÍTICA}

Los proyectos educativos no están ajenos a la vida política de cada país. Si algo caracteriza los últimos 20 años en América Latina es la convulsión política. Semejanzas, diferencias y singularidades van a ser la expresión de estas acciones derivadas de una política educativa común: la eficacia escolar. De múltiples formas el modelo de eficacia fue ampliamente cuestionado por diversos actores y diversas expresiones sociales también en los primeros años del siglo XXI. Sea el movimiento social de los estudiantes secundarios en Chile (los pingüinos), el levantamiento social pidiendo una nueva constitución que se convirtió en un amplio movimiento de masas en ese país en 2019, las luchas de los estudiantes universitarios en Colombia (2018) por garantizar los recursos financieros para las universidades públicas. Los movimientos contra la violencia de género que se hicieron prácticamente globales en 2019 iniciando con el Me too y teniendo un punto relevante con "el violador eres tú". Así como los diversos movimientos de resistencia de los docentes frente a las reformas que impulsan un modelo de evaluación. Estos y otros muchos movimientos sociales, docentes y estudiantiles fueron marcando la necesidad de re-pensar la estructura de las políticas adoptadas por diversos países de la región y en particular contra la política educativa de eficiencia pedagógica implantada en nuestro medio.

En este convulsionado contexto la delincuencia organizada, en particular la que opera en el trasiego de droga, se convirtió en un actor relevante en varias sociedades. Un actor que no sólo siembra terror, muerte y desconcierto; que ya no se limita solamente al trasiego de droga y a promover el consumo entre los niños y jóvenes de nuestras sociedades, sino que ante la pauperización de la sociedad latinoamericana se convirtió en "fuente de empleo" para jóvenes que dada la falta de futuro que encuentran en la sociedad donde viven, ven esta puerta como una opción de obtener algo, a cambio de todos los riesgos que implica.

Ciertamente en esta convulsión social, económica y educativa empezaron también a configurarse otras formas de ascenso al poder político en la región. Se pasó de los golpes de estado realizados por militares en la segunda mitad del siglo XX, a la llegada al poder de diversos actores sociales que ya no respondían a la llamada lógica del mercado. La diversidad de actores políticos y de posiciones políticas que existieron en la región es claro ejemplo de ello, en diversos momentos de cada país obtuvieron el poder político grupos o personas más cercanos a las visiones liberales cercanas al mercado o a las necesidades de su región, en particular la atención a los pobres. El cuestionamiento a la concentración de la riqueza en cada país constituyó un elemento central en las diversas oscilaciones de política que existieron. La OEA empezó a perder el papel que había desempeñado en la región ante la emergencia del merco sur o de la asociación de países del pacífico, varias de sus decisiones fueron menguando su legitimidad en la región, como la intervención en las elecciones en 
Bolivia que desembocaron en la renuncia del presidente de esa nación. Emergieron asociaciones con diversos fines cada una, con un profundo sentido ideológico, pero en donde la tradicional caracterización política derecha/izquierda ha sido insuficiente para identificarlas.

\section{LAS COMUNIDADES ACADÉMICAS ENTORNO A LA EDUCACIÓN Y AL CAMPO DEL CURRÍCULO}

Todo ello explica parcialmente el que podamos reconocer la existencia de diversas comunidades académicas en cada país, las que no necesariamente responden a una misma lógica, ni a un mismo proyecto. Lo educativo, las políticas para este sector también se convirtieron en un espacio claro de lucha ideológica, la bandera de calidad de la educación se enfrentó a los proyectos de formar ciudadanos para comprender las necesidades de su entorno, para resolver los problemas que se encuentran a su alrededor.

El desarrollo de diversas disciplinas tales como la didáctica (o la ausencia de ella en varios países), la psicología educativa (con las diversas vertientes que permanecen en la región desde el socioconstructivismo, la psicología social reconstruida desde el psicoanálisis, así como la perspectiva psicométrica aplicada a la educación), de la sociología con sus diversos clivajes desde la sociología funcionalista, las formas de neomarxismo, así como los pensamientos que tienen una mezcla de perspectivas epistémicas, filosóficas, las posiciones posmodernas y las visiones de teoría de la ciencia ancladas en la búsqueda de teorías; la reconstrucción del sujeto social desde la perspectiva de Foucault, Derrida, entre muchos más, han sido la fuente donde se han ido conformando diversas escuelas y tradiciones en el campo del currículo.

Los autores de referencia se encuentran en el marco de lo que podríamos considerar los grandes aparatos conceptuales, que permiten establecer a nivel internacional diversas formas de interpretación curricular, que han ido ayudando a conformar diversos grupos académicos en torno a la educación y en particular al campo del currículo. De esta manera encontramos los estudios que plantean reconceptualizar el currículo y el método currere (Pinar); la visión de corte más sociológico que busca analizar al currículo como una expresión de una política cultural (Goodson); el empleo de la teoría del discurso en el campo curricular, apoyándose en los planteamientos de Laclau (De Alba, Casimiro); la conceptualización de la educación como espacio de liberación y emancipación social (Giroux, McClaren); el socioconstructivismo en el campo curricular (Coll, Díaz-Barriga Arceo); el currículo como práctica social, en alguna de las vertientes de sus orígenes como la teoría deliberativa del currículo (Schwab) o bien el papel de los docentes en la reconstrucción curricular (Stenhouse), así como la vinculación con los proyectos didácticos (Díaz Barriga). Son algunas de entre múltiples formas de aproximarse a construir una interpretación en el desarrollo de diversas categorías y explicaciones de lo que acontece en el campo.

Algunos académicos han preferido acogerse al uso castellano que Pinar da al campo como una conversación compleja, tema que aparece novedoso en nuestro medio, pero que no necesariamente retoma el sentido que el sentido lingüístico tiene en inglés, pues lo más semejante a conversación compleja en castellano podría ser discusión, diálogo o debate de 
ideas. Ciertamente esta introducción al castellano de este término constituye una forma epocal del momento que estamos viviendo, de hecho reconocemos la castellanización de varios anglisismos tales como "agenciar" (de agency), "gamificar" (de games), "webinar" (una charla o presentación por medios digitales), etc. ${ }^{2}$ Sin embargo, al adoptar en el campo curricular el término conversación compleja en realidad se busca remitir a un autor, Pinar, así como a varios elementos de su conceptualización no sólo al tema de la reconceptualización del campo, sino también a la asunción de otros elementos de su propuesta como el método currere como forma de investigación el pasado y presente del campo, para concebir su futuro.

Todos estos autores mencionados previamente se han constituido en referentes del debate curricular no sólo en la región, sino en cada país, su presencia es más o menos retomada por diversos actores nacionales, en algunos casos como un trabajo casi individual del académico y en otras ocasiones, ciertamente las menos, logrando conformar un grupo que trabaja el tema desde una institución específica o desde la conformación de un seminario de investigación. La diversidad de expresiones que asume la presencia de los actores del campo curricular es una de las dificultades que tenemos para establecer una identificación puntual de la expresión conceptual que tiene el campo del currículo en nuestro medio.

Los procesos de desarrollo del campo curricular van más allá de lo que en un momento Moreira denominó hibridación del campo, mientras Díaz Barriga lo consideró como un mestizaje. Pues con el avance de los estudios del currículo se puede reconocer un proceso de apropiación, adscripción a una corriente o escuela de pensamiento curricular, al mismo tiempo que acontece su reformulación. Esto es, como un efecto de la globalización y de la posibilidad de intercambio que ofrecen las tecnologías digitales, las discusiones y aportaciones de académicos de América Latina, cada vez más refleja influencias del debate internacional y, al mismo tiempo que realizan aportes para el desarrollo del mismo. Lo que Pinar concibe como la etapa de cosmopolitizacion del campo.

\section{DOCUMENTAR LOS GRUPOS DE DISCUSIÓN, UNA TAREA PENDIENTE EN LA REGIÓN}

En América Latina podemos reconocer la existencia de autores y de corrientes que reflejan diversas posiciones o escuelas en el campo curricular. Hay posicionamientos y la mayoría de ellos son muy claros, si bien ya se pueden identificar algunos grupos entorno a una de estas escuelas de pensamiento, no contamos con suficientes elementos para explorar con mayor profundidad la riqueza conceptual que hay en la región. De hecho, podríamos afirmar que para un análisis fino se nos escapa la mayoría de las vertientes por las que el camino curricular se desarrolla en la región.

Quizá si eliminamos la visión dominante de concebir al currículo como planes de estudios, desde la perspectiva de su elaboración, aplicación o valoración, tema en donde existe un grupo muy grande de académicos ante la necesidad institucional de contar con este artefacto (Goodson, 2003). Tema sobre el cual la evidencia es excesiva, lo que no significa que

\footnotetext{
${ }^{2}$ Tema que en estricto sentido no nos debía asustar en un país que adoptó computadora por computer; troca por truck; mientras que en otros de la región se emplea bus por camión de pasajeros, etc.
} 
realmente contenga aportaciones significativas, ni mucho menos que los cambios en los planes y programas de estudio realmente logren los propósitos que enuncia. En los hechos cada reforma a un plan de estudios se constituye en una promesa utópica de los logros que gracias a ella se obtendrían, sin necesidad de modificar necesariamente las formas de relación pedagógica que se establecen en el aula, moviendo el orden de los contenidos, pero tampoco logrando una confrontación entre esa misma jerarquización, para después de determinado tiempo, establecer la necesidad de una nueva reforma que logre lo que ninguna de las anteriores había logrado. Las reformas curriculares viven envueltas en un permanente círculo vicioso y ocupan un tiempo significativo en las formas de estudio del campo curricular en la región.

Muchos académicos han llegado a mimetizar el campo de la investigación curricular, sólo a las estrategias para realizar estos cambios. El tiempo y el esfuerzo que requiere cada reforma agota en sí misma a los grupos y los procesos curriculares. Este rubro enmarca un problema epistémico que en algún momento tendríamos que convertir en objeto de indagación en el campo, dado que en esta tendencia no sólo es dominante, en la mayoría de las ocasiones permanece en un plano exclusivamente formal y, al mismo tiempo, dificulta la indagación rigurosa de lo que constituye el campo curricular

Sin embargo, si regresamos a la necesidad de identificar los grupos que tienen una línea sólida de investigación del curriculum, nos encontramos con un conjunto de dificultades.

La primera dificultad que enfrentamos en esta tarea es la forma colonizada que reproducimos en nuestro trabajo intelectual. Leemos más y buscamos intercambio con académicos del primer mundo, tema que no es cuestionable en sí mismo, a menos que reconozcamos que no mostramos el mismo interés por leer lo que se produce en la región. Alguna investigadora mexicana cuando se le preguntó si acostumbraba a leer lo que se producía en el campo en México, claramente respondió que no era de su interés.

A esta falta de interés hay que agregar la dificultad para acceder a los libros que se producen en las editoriales de la región. La gran mayoría de ellos sólo tiene una escasa circulación local, y en muchas ocasiones, sobre todo cuando se trata de ediciones académicas universitarias, sólo se puede tener acceso en las librerías propias de cada institución. Lo cual hace que realmente sea muy circunstancial el poder identificar un libro de un autor de la región.

En el caso de los artículos podemos reconocer que estamos ante una mejora de la situación, aunque con limitaciones si atendemos al mundo global. Ciertamente se puede acceder a través de las principales bases de datos en castellano (Redalyc, Scielo, Lantindex, IRESIE) de las publicaciones realizadas en revistas científicas. Pero las bases de datos en español o portugués son sólo un pequeño segmento de la producción global, baste con ver el informe que elaboró la UNESCO sobre las Ciencias Sociales en el Mundo. (UNESCO, 2010). Si bien tienen el rasgo de proporcionar el material de acceso libre, lo que no acontece con las otras bases de datos EBSCO, Eric, Academic, Social Science, que representan la producción global de lo que se produce en investigación. 
El impacto de las publicaciones en inglés ha convertido a este idioma en la lengua franca para la comunicación científica, lo que en algún momento dificulta el intercambio entre académicos de América Latina y académicos de todo el globo. Se trata de una realidad que no se puede desconocer, aun cuando bases de datos como Scielo tengan inicialmente en su origen dar visibilidad internacional a lo que se produce en Portugués y posteriormente en castellano y en buena medida han contribuido a ello. Estamos a los que Brunner \& Zalazar (2001) estudiaron como la falta de visibilidad de la investigación latinoamericana en educación (2011). Estos autores identificaron la diferencia que hay entre el material que se ubica en google académico (scholar google) y las bases de datos tales como SCOPUS, ISI Thompson, entre otras.

Esta situación acentúa la actitud colonial que existe en algunas de nuestras comunidades académicas, sea desde el punto de vista de inclusión de esta visión o de rechazo a lo producido en otro idioma. Para algunos miembros de la comunidad académica trabajar y escribir en el interés ético y político de que los resultados de la investigación impacten la realidad educativa de la región demanda publicar en castellano o portugués, tema que entra en conflicto con la visibilidad e impacto del trabajo académico en nuestro medio en este momento.

En este contexto podemos afirmar que existe un desconocimiento, aunado a una ausencia de investigación sistemática sobre los rasgos que representan los principales grupos de investigación curricular en la región. Ante esta situación consideramos que en este momento se pueden considerar tres textos significativos para avanzar hacia la realización de esta tarea.

Una de las primeras producciones que buscaron documentar la situación del campo del curriculum en la región es la realizada por Díaz-Barriga \& García Garduño (2014)³, el libro es el resultado de un proyecto para identificar los mecanismos a través de los cuáles se incorporó el tema curricular en la región y la reacción que hubo frente a ello. El estudio se realizó mediante la invitación a diversos especialistas latinoamericanos para documentar la inserción curricular en sus países y los procesos que se generaron a partir de los años sesenta y setenta del siglo pasado. Los mecanismos que se dieron en la región para la difusión de esta disciplina como un acto de colonización cultural, imperialismo cultural lo denominó Carnoy (1977). Es interesante reconocer la forma como el tema curricular y otros que forman parte de la pedagogía estadounidense desarrollada a principios del siglo XX se difundieron en América Latina durante estos años, en particular la llamada teoría de objetivos de aprendizaje. Lo primero que se encontró fue documentar los diversos mecanismos que se emplearon en la región. En unos países enviaron a académicos a formarse en los EEUU para que regresaran a aplicar lo aprendido en su país; otros decidieron contratar profesores estadounidenses para que desarrollaran talleres en los Ministerios de Educación; y, unos más

\footnotetext{
${ }^{3}$ Si bien reconocemos que al principio de la década de los años noventa del siglo pasado Alicia de Alba (1995) en el marco del proyecto Curriculum y siglo XXI coordinó la publicación sobre el curriculum universitario México Argentina. Mientras que Díaz Barriga coordinó el grupo que trabajó la Red Iberoamericana de Investigadores sobre el Curriculum (RIIC)
} 
acudieron a los centros de formación de profesores universitarios para desde las Universidades trabajar con estos conceptos. Lo que se generalizó fue la perspectiva técnica del currículo fincada fundamentalmente en el pensamiento de Bloom, Tyler y Mager.

Si bien, los modelos pedagógicos derivados de la teoría curricular se fueron aplicando en los diversos países el mestizaje o hibridación de las teorías se fue gestando de manera muy rápida en la región, llegando a propuestas como el sistema modular por objetos de transformación (por problemas en el lenguaje que hoy conocemos) (Díaz Barriga, et al 1986) que se construyó sin ninguna referencia a estos autores. Dos impactos tuvo la incorporación de esta visión pedagógica en la región, en primer término contribuyó a una transformación del plan de los estudios concebidos sólo como una lista de materias en una estructura anual o semestral, a la elaboración de un documento curricular donde se especificara con mayor claridad el proyecto de formación y sus fundamentos, perfil de egreso, así como una descripción detallada de los contenidos de cada una de las materias, aunque también se convirtió en el espacio para formular propuestas curriculares integradas, construidas a partir de módulos generados con relación a un problema (Díaz Barriga, en 2020). Mientras que, en segundo lugar, la aplicación de la teoría de objetivos de aprendizaje inició una ruta de pensar el trabajo que se realiza en el aula en función de lo que el alumno tiene que hacer y no de los contenidos que el docente tiene que enseñar, esto es, abandonar una visión enciclopédica de la enseñanza. Una lucha, por cierto, que aún no se gana, pues a partir de la primera década del siglo XXI el planteamiento de trabajar por competencias, en el fondo vuelve a establecer el tema de pensar más en el aprendizaje que en la enseñanza.

Los otros trabajos que se han realizado en la región son propiamente estudios comparados realizados sobre la situación del campo curricular en algunos países. En esta línea encontramos dos trabajos, Morelli, (2016) y De Alba y Casimiro (2014 y 2015).

Iniciamos el planteamiento a partir del trabajo de Morelli (2016) quien hace una comparación mucho más analítica sobre la situación de la producción curricular en México y Argentina. Un documento que parte de una rigurosa conceptualización, a partir de la cual recupera una importante cantidad de literatura específica del campo del currículo en ambos países.

La autora parte de clasificar las perspectivas de los trabajos identificados en ambos países en dos grandes tendencias a) los autores vinculados al pensamiento de la modernidad donde ubica el pensamiento de Díaz Barriga particularmente, b) frente a los que trabajan el pensamiento posmoderno, poscrítico, y retoman la teoría del discurso de Laclau, donde ubica al pensamiento de Pinar, de Alba, Orozco, Gallardo, entre otros. Quizá, y esa puede ser una afirmación que tiene el riesgo de ser superficial, la clasificación, esta clasificación tenga en sí misma algún elemento de lo que he denominado la colonización en el campo curricular. Pues si Morelli concibe a los autores del pensamiento moderno como aquellos que están preocupados por lo que acontece con relación a sus sistemas educativos, esto es, autores que han asumido un compromiso ético-político con su realidad pedagógica, entonces una consecuencia sería que los vinculados al pensamiento posmoderno y poscrítico en realidad reflejan el interés (en sentido habermasiano del término) de discutir de manera intelectual con académicos del globo. Goodson (2003) cuestiona el énfasis que colocan en lo individual 
estos pensamientos que tienen una condición pos (crítica, moderna, estructuralista) expresando que significan "una retirada del mundo académico a un mundo intelectualmente esotérico" que pierde la perspectiva de anclarse en las políticas curriculares, que se desarrollan en detrimento del conocimiento de lo político y de lo social, en "un narcisismo y una autoindulgencia que ha ganado terreno en los debates curriculares" (Goodson 2003). La clasificación que hace Morelli (2016) por demás resulta válida, si bien la autora no logra percibir las diversas implicaciones que ello conlleva.

Una interrogante que surge a través de la lectura de los textos de quienes trabajan el pensamiento poscrítico y posmoderno requiere analizar hasta donde esta visión complica el lenguaje y su discurso, para dar cuenta de fenómenos que podrían enunciar de una manera más simple. En el fondo lo que ambos grupos de autores intentamos, es dar cuenta de la complejidad del momento social que vivimos y su impacto en los diferentes procesos educativos; la diversidad de intereses, posibilidades, formas de acceso, expresiones culturales y económicas de los diversos actores de la educación; las presiones hegemónicas y contrahegemónicas que se viven en el campo del currículo y de la educación en general. Es necesario preguntarse en este momento hasta dónde es necesario cuidar el rigor conceptual, los documentos escritos, para inscribirlos en una tradición moderna o posmoderna; habermasiana o de la teoría crítica, para dar cuenta de una realidad que tenemos enfrente y, frente a la cual tenemos la obligación de identificarla, reconocerla, analizarla y hasta donde sea factible trabajar por mejorarla.

Morelli (2016) invita a realizar otra discusión con relación al término internacionalización del currículo. Plantea que Diaz Barriga considera que este fenómeno aconteció en la década de los años setenta del siglo pasado con la difusión del pensamiento curricular estadounidense, la traducción masiva con distribución gratuita de ciertos autores. Mientras que para Pinar la internacionalización del currículo se debe a que en los años noventa del siglo pasado, ya se pueden identificar grupos en diversas regiones del mundo que estudian el campo curricular y han generado la posibilidad de intercambiar y debatir sus aportes entre ellos. Una distinción en la diferencia del uso del término que en el fondo responde a la manera como se concibe internacionalización. Lo que no significa negar el papel que ha tenido Pinar en el conjunto de su obra para desarrollar el campo curricular, su noción de currere que lo lleva a pensar que ahí se finca un método de trabajo en el campo y su planteamiento de la necesidad de reconceptualizar el campo. Ciertamente el pensamiento de Pinar es bastante marginal en los debates que se realizan en América Latina. Lo cual, incluso se puede observar en la dificultad para encontrar traducciones de sus textos al castellano o al portugués. Reconociendo, aunque esto no es objeto del análisis que realiza Morelli (2016) que los países donde el pensamiento de Pinar ha tenido mayor impacto son Brasil, México y Argentina.

Sin embargo, con independencia de la forma como interpretemos la internacionalización del campo del currículo, lo que es un hecho, es que en la conformación de la educación básica y de la formación profesional existieron influencias internacionales que se fueron manifestando a través de la consolidación de los sistemas educativos de los distintos estados nacionales, a lo largo del siglo XIX. 
Entonces la pregunta que cabe hacerse es cuál fue el sentido de la expansión del campo curricular en la región. Quizá la respuesta más clara la ofrezca el estudio que hace Tröhler (2017), cuando plantea que el sentido de esta internacionalización fue incorporar una forma de ver el trabajo escolar, la enseñanza a través de una visión mediada por el mercado. Incorporar la lógica de la eficacia escolar como un elemento nodal del trabajo escolar. La escuela para la vida, pero no para la vida del sujeto alumno, en el plano de su desarrollo y potencial personal, sino la escuela para la vida ciudadana y productiva. Así cobran sentido las teorías gerenciales y productivistas que han acompañado el debate curricular sea a través de conceptos que ven la adquisición de comportamientos como aprendizaje, el desarrollo de competencias como habilidades para la vida.

\section{CONSIDERACIONES FINALES}

El campo del currículo actualmente es complejo, tiene varias vertientes y escuelas de pensamiento. En América Latina no se trabaja necesariamente a partir de todas estas perspectivas de pensamiento. Ciertamente hay un conjunto de autores que suelen manejarse con mayor familiaridad, los vinculados a los temas del currículo formal y al currículo real tienen preeminencia sobre las otras perspectivas. En ocasiones su perspectiva se llega a constituir en un obstáculo epistemológico en términos de Bachelard, para avanzar hacia otras rutas. Por diversas razones, los autores de una visión crítica del currículo suelen tener también cierta presencia en los debates que se dan en la región.

En algunos países ya se pueden identificar grupos de académicos que se forman y estudian en alguna corriente curricular diferente a la dominante, mientras que en la mayoría de los países latinoamericanos todavía no se observa con claridad la conformación de estos grupos, sino que se pueden detectar solo trabajos que reflejan el proceso individual de algún académico.

Existe una dificultad real para impulsar un intercambio entre académicos que trabajen temas curriculares en América Latina, ello se debe a varias circunstancias, la que tiene mayor peso es una actitud colonizada que lleva a buscar y leer autores fundamentalmente del primer mundo, a buscar realizar un intercambio con ellos, parte de esta situación también se debe el proceso de internacionalización y evolución cosmopolita del debate curricular. En sí mismo esto no sería cuestionable si no fuera acompañado por una actitud de desinterés por lo que se produce en la región. Los autores latinoamericanos no se leen entre latinoamericanos esa es una realidad que pesa sobre este campo de investigación y quizá sobre otros también.

A esta actitud se suma una dificultad para tener acceso a las publicaciones de textos en América Latina. Reconociendo que el acceso que las bases de datos brindan a lo publicado en revistas en castellano o en portugués, el tema es mucho más complicado cuando se trata de libros publicados, en general por editoriales universitarias, cuyos materiales sólo se pueden adquirir en las librerías de estas instituciones y, que en general, su publicación es desconocida por la comunidad académica. 
A ello hay que agregar que la investigación que busque dar cuenta de lo que se publica en América Latina es prácticamente incipiente. Existe un pequeño avance de intercambio en los congresos, pero este intercambio no es suficiente para considerarlo como investigaciones sistemáticas sobre el tema.

Si bien existen ya grupos conformados y académicos que tienen una clara línea de investigación en el campo del curriculum, así como programas de posgrado específicamente vinculados a este ámbito e incluso una significativa cantidad de cursos sobre el tema en los posgrados. Este trabajo es aún insuficiente para que en ellos se trabaje en relación a la perspectiva curricular construida desde América Latina.

En el trabajo también mostramos el escaso impacto que tienen algunos autores del campo del currículo que han conformado ya una línea de investigación en otros países e incluso la nula presencia que otros autores tienen en la región.

Finalmente, el reto es incrementar los análisis e investigaciones que se hacen en relación a la producción curricular en América Latina, lo que demanda fortalecer los grupos que existen o se están conformando en cada país. Asumir una actitud diferente frente a la producción latinoamericana abriendo espacios para intercambio, difusión y discusión de lo que se produce en la región. Ciertamente que el pensamiento curricular latinoamericano no puede, ni debe excluirse de las escuelas y corrientes de pensamiento global que existen en este momento, la escritura y divulgación en inglés sigue siendo un reto de la producción latinoamericana, pero sin descuidar el atender y enfrentar los problemas educativos de nuestros países.

\section{BIBLIOGRAFÍA}

Alba, A. de (1991) Evaluación curricular. Conformación conceptual del campo. México. CESUUNAM.

Alba, A. De (1995) Panorama del curriculum universitario 1970-1990. México-Argentina. México, Cuadernos del CESU. CESU. UNAM

Brunner, J. F. Salazar (2011) La investigación educacional en iberoamerica: entre la invisibilidad y la medición, en Revisita Magis, Bogotá, no 4, Vol9 Universidad Javeriana pp 559-575 [https://revistas.javeriana.edu.co/index.php/MAGIS/search/search]

Carnoy, M (1977) La educación como imperialismo cultural. México, Siglo XXI

Díaz Barriga, A. D. Martínez, R. Reygadas, G. Villaseñor (1989) Práctica docente y diseño curricular (Un estudio exploratorio en la UAM-Xochimilco). México, Universidad Autónoma Metropolitana-Xochimilco-Centro de Estudios sobre la Universidad (CESU) UNAM. 
Díaz-Barriga, A. (2009), "A avaliação na educação mexicana. Excesso de pro-gramas e ausência da dimensão pedagógica", Sisifo, Revista de Ciências da Educação,núm. 9, mayo-agosto, en [isifo.fpce.ul.pt/pdfs/Re-vista 9 PT d2.pdf], consultado el 25 de mayo de 2012.

Díaz Barriga, A (2020) "De la integración curricular a las políticas de innovación en la educación superior mexicana" en revista Perfiles Educativos, vol 42, no 169, México, Instituto de Investigaciones sobre la Universidad y la Educación. UNAM [http://perfileseducativos.unam.mx/iisue_pe/index.php/perfiles/article/view/5947 8] consultado 24 de septiembre de 2020

Díaz-Barriga, A. J García-Garduño (Coords) (2014) Desarrollo del currriculum en América Latina. Buenos Aires. Miño y Dávila-Universidad Autónoma de Tlaxcala.

El-Kalwas, Elaine (2001). Acreditation in USA: Origins, Developments and Future Prospects, París, UNESCO/International Institute for Educational Planning. [https://unesdoc.unesco.org/ark:/48223/pf0000129295]

Goodson, I. (2003) Estudio del curriculum: casos y métodos. Buenos Aires, Amorrortu.

Lewy, A (Coord) (1976) Manual para la evaluación formativa del currículo. Bogotá. VoluntadUNESCO

Horlacher, R (2015) Bildung, la formación. Octaedro, Barcelona

Morelli, S (2016) Las tensiones del curriculum Debates político-educativoss en México y Argentina. Buenos Aires, Miña y Dávila

Ruíz, E. (2001) Propuesta de un modelo de evaluación curricularpara el nivel superior. Orientación cualitativa. En Cuadernos del CESU, México, Centro de Estudios sobre la Universidad. UNAM.

UNESCO (2011) Informe las ciencias sociales en el mundo. (Versión en Español de Foro Consultivo y Tecnológico. México) Original en inglés 2010 World Social Science Report 2010: Knowledge Divides. En [http://www.foroconsultivo.org.mx/libros_editados/informe_sobre_las_ciencias_soc iales_en_el_mundo.pdf]

Valenzuela, A (2005) Diseño y validez de una propusta de evaluación curricular en la educación superior. México, Uniiversidad Anáhuac. Tesis de doctorado en educación.

Tröhler, D (2017) “La historia del currículum como camino real a la investigación educativa internacional. Historia, perspectivas, beneficios y dificultades" en revista Profesorado. Revista de curriculum y formación del profesorado. Vol 21, no 1, Universidad de Granada, Granada. pp 202-232 